\title{
LOCALLY CONVEX QUASI C*-ALGEBRAS AND NONCOMMUTATIVE INTEGRATION
}

\author{
CAMILLO TRAPANI AND SALVATORE TRIOLO
}

\begin{abstract}
In this paper we continue the analysis undertaken in a series of previous papers on structures arising as completions of $\mathrm{C}^{*}$-algebras under topologies coarser that their norm and we focus our attention on the so-called locally convex quasi $C^{*}$ algebras. We show, in particular, that any strongly *-semisimple locally convex quasi $\mathrm{C}^{*}$-algebra $\left(\mathfrak{X}, \mathfrak{A}_{0}\right)$, can be represented in a class of noncommutative local $L^{2}$-spaces.
\end{abstract}

\section{INTRODUCTION}

The completion $\mathfrak{X}$ of a $\mathrm{C}^{*}$-algebra $\mathfrak{A}_{0}$, with respect to a norm weaker than the $\mathrm{C}^{*}$ norm provides a mathematical framework where discussing certain quantum physical systems for which the usual algebraic approach made in terms of $\mathrm{C}^{*}$-algebras revealed to be insufficient.

First of all, $\mathfrak{X}$ is a Banach $\mathfrak{A}_{0}$-module and becomes a quasi *-algebra if $\mathfrak{X}$ carries an involution which extends the involution $*$ of $\mathfrak{A}_{0}$. This structure has been called a proper $C Q^{*}$-algebra in a series of papers [4]-[10, 22]-221] to that we refer for a detailed analysis. On the other hand, if $\mathfrak{X}$ is endowed with an isometric involution different from that of $\mathfrak{A}_{0}$, then the structure becomes more involved.

$\mathrm{CQ}^{*}$-algebras are examples of more general structures called locally convex quasi $C^{*}$-algebras $\left[3\right.$. They are obtained by completing a $\mathrm{C}^{*}$-algebra with respect to a new locally convex topology $\tau$ on $\mathfrak{A}_{0}$ compatible with the corresponding $\|\cdot\|$-topology. Under certain conditions on $\tau$ a quasi $*$-subalgebra $\mathfrak{A}$ of the completion $\widetilde{\mathfrak{A}_{0}}[\tau]$ is a locally convex quasi *-algebra which is named locally convex quasi $\mathrm{C}^{*}$-algebra.

In [9] quasi *-algebras of measurable and/or integrable operators (in the sense of Segal [19], 27] and Nelson [17]) were examined in detail and it was proved that any *-semisimple $\mathrm{CQ}^{*}$-algebra can be realized as a $\mathrm{CQ}^{*}$-algebra of measurable operators, with the help of a particular class of positive bounded sesquilinear forms on $\mathfrak{X}$.

In this paper, after a short overview of the main results obtained on this subject, we continue our study of locally convex quasi $\mathrm{C}^{*}$-algebras and we generalize the results obtained in 9] for proper $\mathrm{CQ}^{*}$ - algebras to these structures.

The main question we pose now in the present paper is the following: given a $*_{-}$ semisimple locally convex quasi $\mathrm{C}^{*}$-algebras $\left(\mathfrak{X}, \mathfrak{A}_{0}\right)$ and the universal *-representation of $\mathfrak{A}_{0}$, defined via the Gelfand-Naimark theorem, can $\mathfrak{X}$ be realized as a locally convex quasi $\mathrm{C}^{*}$-algebra of operators of type $L^{2}$ ?

2000 Mathematics Subject Classification. Primary 46L08; Secondary 46L51, 47L60 .

Key words and phrases. Banach $\mathrm{C}^{*}$-modules, Noncommutative integration, Partial algebras of operators. 
The paper is organized as follows. We begin with giving a short overview of noncommutative $L^{p}$-spaces (constructed starting from a von Neumann algebra $\mathfrak{M}$ and a normal, semifinite, faithful trace $\varphi$ on $\mathfrak{M}$ ), considered as $\mathrm{CQ}^{*}$-algebras. We also introduce the noncommutative $L_{\text {loc }}^{p}$-space constructed on a von Neumann algebra possessing a family of mutually orthogonal central projections whose sum is the identity operator. We show that $\left(L_{\text {loc }}^{p}(\varphi), \mathfrak{M}\right)$ is a locally convex quasi $\mathrm{C}^{*}$-algebra.

Finally we give some results on the structure of locally convex quasi $\mathrm{C}^{*}$-algebras: we prove that any locally convex quasi $\mathrm{C}^{*}$-algebra $\left(\mathfrak{X}, \mathfrak{A}_{0}\right)$ possessing a sufficient family of bounded positive tracial sesquilinear forms can be continuously embedded into a locally convex quasi $\mathrm{C}^{*}$-algebras of measurable operators of the type $\left(L_{\mathrm{loc}}^{2}(\varphi), \mathfrak{M}\right)$.

1.1. Definitions and results on noncommutative measure. The following basic definitions and results on noncommutative measure theory and integration are needed in what follows. Let $\mathfrak{M}$ be a von Neumann algebra on a Hilbert space $\mathcal{H}$ and $\varphi$ a normal faithful semifinite trace defined on $\mathfrak{M}_{+}$.

Put

$$
\mathcal{J}=\{X \in \mathfrak{M}: \varphi(|X|)<\infty\}
$$

$\mathcal{J}$ is a $*$-ideal of $\mathfrak{M}$.

Let $P \in \operatorname{Proj}(\mathfrak{M})$, the lattice of projections of $\mathfrak{M}$. Two projections $P, Q \in \operatorname{Proj}(\mathfrak{M})$ are called equivalent, $P \sim Q$ if there is a $U \in \mathfrak{M}$ with $U^{*} U=P$ and $U U^{*}=Q$. We say that $P \prec Q$ in the case where $P$ is equivalent to a subprojection of $Q$.

A projection $P$ of a von Neumann algebra $\mathfrak{M}$ is said to be finite if $P \sim Q \leq P$ implies $P=Q$. A projection $P \in \mathfrak{M}$ is said to be purely infinite if there is no nonzero finite projection $Q \preceq P$ in $\mathfrak{M}$. A von Neumann algebra $\mathfrak{M}$ is said to be finite (respectively, purely infinite) if the identity operator $\mathbb{I}$ is finite (respectively, purely infinite).

We say that $P$ is $\varphi$-finite if $P \in \mathcal{J}$. Any $\varphi$-finite projection is finite.

In what follows we will need the following result (see, [15, Vol. IV, Ex. 6.9.12]) that we state as a lemma.

Lemma 1.1. Let $\mathfrak{M}$ be a von Neumann algebra on a Hilbert space $\mathcal{H}$ and $\varphi$ a normal faithful semifinite trace defined on $\mathfrak{M}_{+}$. There is an orthogonal family $\left\{Q_{j} ; j \in J\right\}$ of nonzero central projections in $\mathfrak{M}$ such that $\bigvee_{j \in J} Q_{j}=\mathbb{I}$ and each $Q_{j}$ is the sum of an orthogonal family of mutually equivalent finite projections in $\mathfrak{M}$.

A vector subspace $\mathcal{D}$ of $\mathcal{H}$ is said to be strongly dense ( resp., strongly $\varphi$-dense) if

- $U^{\prime} \mathcal{D} \subset \mathcal{D}$ for any unitary $U^{\prime}$ in $\mathfrak{M}^{\prime}$

- there exists a sequence $P_{n} \in \operatorname{Proj}(\mathfrak{M}): P_{n} \mathcal{H} \subset \mathcal{D}, P_{n}^{\perp} \downarrow 0$ and $P_{n}^{\perp}$ is a finite projection (resp., $\varphi\left(P_{n}^{\perp}\right)<\infty$ ).

Clearly, every strongly $\varphi$-dense domain is strongly dense.

Throughout this paper, when we say that an operator $T$ is affiliated with a von Neumann algebra, written $T \eta \mathfrak{M}$, we always mean that $T$ is closed, densely defined and $T U \supseteq U T$ for every unitary operator $U \in \mathfrak{M}^{\prime}$.

An operator $T \eta \mathfrak{M}$ is called

- measurable (with respect to $\mathfrak{M}$ ) if its domain $D(T)$ is strongly dense; 
- $\varphi$-measurable if its domain $D(T)$ is strongly $\varphi$-dense.

From the definition itself it follows that, if $T$ is $\varphi$-measurable, then there exists $P \in \operatorname{Proj}(\mathfrak{M})$ such that $T P$ is bounded and $\varphi\left(P^{\perp}\right)<\infty$.

We remind that any operator affiliated with a finite von Neumann algebra is measurable [19, Cor. 4.1] but it is not necessarily $\varphi$-measurable.

Remark 1.2. The following statements will be used later.

(i) Let $T \eta \mathfrak{M}$ and $Q \in \mathfrak{M}$. If $D(T Q)=\{\xi \in \mathcal{H}: Q \xi \in D(T)\}$ is dense in $\mathcal{H}$, then $T Q \eta \mathfrak{M}$.

(ii) If $Q \in \operatorname{Proj}(\mathfrak{M})$, then $Q \mathfrak{M} Q=\left\{Q X Q \uparrow_{Q \mathcal{H}} ; X \in \mathfrak{M}\right\}$ is a von Neumann algebra on the Hilbert space $Q \mathcal{H}$; moreover $(Q \mathfrak{M} Q)^{\prime}=Q \mathfrak{M}^{\prime} Q$. If $T \eta \mathfrak{M}$ and $Q \in \mathfrak{M}$ and $D(T Q)=\{\xi \in \mathcal{H}: Q \xi \in D(T)\}$ is dense in $\mathcal{H}$, then $Q T Q \eta Q \mathfrak{M} Q$.

Let $\mathfrak{M}$ be a von Neumann algebra on a Hilbert space $\mathcal{H}$ and $\varphi$ a normal faithful semifinite trace defined on $\mathfrak{M}_{+}$. For each $p \geq 1$, let

$$
\mathcal{J}_{p}=\left\{X \in \mathfrak{M}: \varphi\left(|X|^{p}\right)<\infty\right\} \text {. }
$$

Then $\mathcal{J}_{p}$ is a ${ }^{*}$-ideal of $\mathfrak{M}$. Following [17, we denote with $L^{p}(\varphi)$ the Banach space completion of $\mathcal{J}_{p}$ with respect to the norm

$$
\|X\|_{p, \varphi}:=\varphi\left(|X|^{p}\right)^{1 / p}, \quad X \in \mathcal{J}_{p} .
$$

One usually defines $L^{\infty}(\varphi):=\mathfrak{M}$. Thus, if $\varphi$ is a finite trace, then $L^{\infty}(\varphi) \subset L^{p}(\varphi)$ for every $p \geq 1$. As shown in [17], if $X \in L^{p}(\varphi)$, then $X$ is a measurable operator.

If $A$ is a measurable operator and $A \geq 0$, one defines the integral of $A$ by

$$
\mu(A)=\sup \left\{\varphi(X): 0 \leq X \leq A, X \in \mathcal{J}_{1}\right\} .
$$

Then the space $L^{p}(\varphi)$ can also be defined [17] as the space of all measurable operators $A$ such that $\mu\left(|A|^{p}\right)<\infty$.

The integral of an element $A \in L^{p}(\varphi)$ can be defined, in obvious way, taking into account that any measurable operator $A$ can be decomposed as $A=B_{+}-B_{-}+i C_{+}-$ $i C_{-}$, where $B=\frac{A+A^{*}}{2}$ and $C=\frac{A-A^{*}}{2 i}$ and $B_{+}, B_{-}$(resp. $\left.C_{+}, C_{-}\right)$are the positive and negative parts of $B$ (resp. $C$ ).

1.2. Locally convex quasi $\mathbf{C}^{*}$-algebras. In what follows we recall some definitions and facts needed in the sequel.

Definition 1.3. Let $\mathfrak{X}$ be a complex vector space and $\mathfrak{A}_{0}$ a ${ }^{*}$-algebra contained in $\mathfrak{X}$. $\mathfrak{X}$ is said a quasi ${ }^{*}$-algebra with distinguished ${ }^{*}$-algebra $\mathfrak{A}_{0}$ (or, simply, over $\mathfrak{A}_{0}$ ) if

(i) the multiplication of $\mathfrak{A}_{0}$ is extended on $\mathfrak{X}$ as follows: the correspondences

$\mathfrak{X} \times \mathfrak{A}_{0} \rightarrow \mathfrak{A}:(a, x) \mapsto a x$ (left multiplication of $x$ by $a$ ) and

$\mathfrak{A}_{0} \times \mathfrak{X} \rightarrow \mathfrak{A}:(x, a) \mapsto x a$ (right multiplication of $x$ by $a$ ) are always defined and are bilinear;

(ii) $x_{1}\left(x_{2} a\right)=\left(x_{1} x_{2}\right) a,\left(a x_{1}\right) x_{2}=a\left(x_{1} x_{2}\right)$ and $x_{1}\left(a x_{2}\right)=\left(x_{1} a\right) x_{2}$, for all $x_{1}, x_{2} \in$ $\mathfrak{A}_{0}$ and $a \in \mathfrak{X}$

(iii) the involution $*$ of $\mathfrak{A}_{0}$ is extended on $\mathfrak{X}$, denoted also by $*$, and satisfies $(a x)^{*}=$ $x^{*} a^{*}$ and $(x a)^{*}=a^{*} x^{*}$, for all $x \in \mathfrak{A}_{0}$ and $a \in \mathfrak{X}$. 
Thus a quasi *algebra 18 is a couple $\left(\mathfrak{X}, \mathfrak{A}_{0}\right)$, where $\mathfrak{X}$ is a vector space with involution ${ }^{*}, \mathfrak{A}_{0}$ is a ${ }^{*}$-algebra and a vector subspace of $\mathfrak{X}$ and $\mathfrak{X}$ is an $\mathfrak{A}_{0}$-bimodule whose module operations and involution extend those of $\mathfrak{A}_{0}$. The unit of $\left(\mathfrak{X}, \mathfrak{A}_{0}\right)$ is an element $e \in \mathfrak{A}_{0}$ such that $x e=e x=x$, for every $x \in \mathfrak{X}$.

A quasi *-algebra $\left(\mathfrak{X}, \mathfrak{A}_{0}\right)$ is said to be locally convex if $\mathfrak{X}$ is endowed with a topology $\tau$ which makes $\mathfrak{X}$ a locally convex space and such that the involution $a \mapsto a^{*}$ and the multiplications $a \mapsto a b, a \mapsto b a, b \in \mathfrak{A}_{0}$, are continuous. If $\tau$ is a norm topology and the involution is isometric with respect to the norm, we say that $\left(\mathfrak{X}, \mathfrak{A}_{0}\right)$ is a normed quasi ${ }^{*}$-algebra and, if it is complete, we say it is a Banach quasi*-algebra.

Let $\mathfrak{A}_{0}\left[\|\cdot\|_{0}\right]$ be a $\mathrm{C}^{*}$-algebra. We shall use the symbol $\|\cdot\|_{0}$ of the $\mathrm{C}^{*}$-norm to denote the corresponding topology. Suppose that $\tau$ is a topology on $\mathfrak{A}_{0}$ such that $\mathfrak{A}_{0}[\tau]$ is a locally convex $*$-algebra. Then, the topologies $\tau,\|\cdot\|_{0}$ on $\mathfrak{A}_{0}$ are compatible whenever each Cauchy net in both topologies that converges with respect to one of them, also converges with respect to the other one.

Under certain conditions on $\tau$ a quasi $*$-subalgebra $\mathfrak{A}$ of the quasi $*$-algebra $\mathfrak{X}=\widetilde{\mathfrak{A}_{0}}[\tau]$ over $\mathfrak{A}_{0}$ is named locally convex quasi $C^{*}$-algebra. More precisely, let $\left\{p_{\lambda}\right\}_{\lambda \in \Lambda}$ be a directed family of seminorms defining the topology $\tau$. Suppose that $\tau$ is compatible with $\|\cdot\|_{0}$ and that it has the following properties:

- $\left(\mathrm{T}_{1}\right) \mathfrak{A}_{0}[\tau]$ is a locally convex *-algebra with separately continuous multiplication.

- $\left(\mathrm{T}_{2}\right) \tau \preceq\|\cdot\|_{0}$.

Then, the identity map $\mathfrak{A}_{0}\left[\|\cdot\|_{0}\right] \rightarrow \mathfrak{A}_{0}[\tau]$ extends to a continuous $*$-linear map $\mathfrak{A}_{0}\left[\|\cdot\|_{0}\right] \rightarrow \widetilde{\mathfrak{A}_{0}}[\tau]$. Since $\tau,\|\cdot\|_{0}$ are compatible, the $\mathrm{C}^{*}$-algebra $\mathfrak{A}_{0}\left[\|\cdot\|_{0}\right]$ can be regarded as embedded into $\widetilde{\mathfrak{A}_{0}}[\tau]$. It is easily shown that $\widetilde{\mathfrak{A}_{0}}[\tau]$ is a quasi $*$-algebra over $\mathfrak{A}_{0}$ (cf. [13, Section 3]).

We denote by $\left(\mathfrak{A}_{0}\right)_{+}$the set of all positive elements of the $\mathrm{C}^{*}$-algebra $\mathfrak{A}_{0}\left[\|\cdot\|_{0}\right]$.

Further, we employ the following two extra conditions $\left(\mathrm{T}_{3}\right),\left(\mathrm{T}_{4}\right)$ for the locally convex topology $\tau$ on $\mathfrak{A}_{0}$

- $\left(\mathrm{T}_{3}\right)$ For each $\lambda \in \Lambda$, there exists $\lambda^{\prime} \in \Lambda$ such that

$$
p_{\lambda}(x y) \leq\|x\|_{0} p_{\lambda^{\prime}}(y), \forall x, y \in \mathfrak{A}_{0} \text { with } x y=y x ;
$$

- $\left(\mathrm{T}_{4}\right)$ The set $\mathcal{U}\left(\mathfrak{A}_{0}\right)_{+}:=\left\{x \in\left(\mathfrak{A}_{0}\right)_{+}:\|x\|_{0} \leq 1\right\}$ is $\tau$-closed.

Definition 1.4. By a locally convex quasi $C^{*}$-algebra over $\mathfrak{A}_{0}$, (see [3]), we mean any quasi *-subalgebra $\mathfrak{A}$ of the locally convex quasi $*$-algebra $\mathfrak{X}=\widetilde{\mathfrak{A}_{0}}[\tau]$ over $\mathfrak{A}_{0}$, where $\mathfrak{A}_{0}\left[\|\cdot\|_{0}\right]$ is a $\mathrm{C}^{*}$-algebra with identity $e$ and $\tau$ a locally convex topology on $\mathfrak{A}_{0}$, defined by a directed family of seminorms $\left\{p_{\lambda}\right\}_{\lambda \in \Lambda}$, satisfying the conditions $\left(\mathrm{T}_{1}\right)-\left(\mathrm{T}_{4}\right)$.

The following examples have been discussed in [3].

Example 1.5. [ $\mathrm{CQ}^{*}$-algebras] Let $\mathfrak{A}_{0}$ be a $C^{*}$-algebra, with norm $\|\cdot\|$ and involution *. Let $\|\cdot\|_{1}$ be a norm on $\mathfrak{A}_{0}$, weaker than $\|\cdot\|$ and such that, for every $a, b \in \mathfrak{A}$

(i) $\|a b\|_{1} \leq\|a\|_{1}\|b\|$,

(ii) $\left\|a^{*}\right\|_{1}=\|a\|_{1}$. 
Let $\mathfrak{X}$ denote the $\|\cdot\|_{1}$-completion of $\mathfrak{A}_{0}$; then 1 the couple $\left(\mathfrak{X}, \mathfrak{A}_{0}\right)$ is called a $C Q^{*}$ algebra. Every $\mathrm{CQ}^{*}$-algebra is a locally convex quasi $\mathrm{C}^{*}$-algebra.

Example 1.6. The space $L^{p}([0,1])$, with $1 \leq p<+\infty$ is a Banach $L^{\infty}([0,1])$-bimodule. The couple $\left(L^{p}([0,1]), L^{\infty}([0,1])\right.$ may be regarded as a $\mathrm{CQ}^{*}$-algebra thus a locally convex quasi $\mathrm{C}^{*}$-algebra over $L^{\infty}([0,1])$.

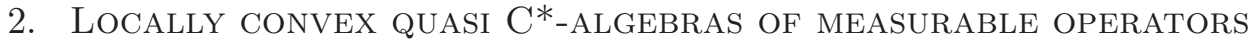

Let $\mathfrak{M}$ be a von Neumann algebra on a Hilbert space $\mathcal{H}$ and $\varphi$ a normal faithful semifinite trace on $\mathfrak{M}_{+}$, then, as shown in [9], $\left(L^{p}(\varphi), L^{\infty}(\varphi) \cap L^{p}(\varphi)\right)$ is a Banach quasi ${ }^{*}$-algebra and if $\varphi$ is a finite trace, $\left(L^{p}(\varphi), \mathfrak{M}\right)$ is a $\mathrm{CQ}^{*}$-algebra.

In analogy to 9 we consider the following two sets of sesquilinear forms enjoying certain invariance properties.

Definition 2.1. Let $\left(\mathfrak{X}, \mathfrak{A}_{0}\right)$ be a locally convex quasi $\mathrm{C}^{*}$-algebra with unit $e$. We denote by $\mathcal{S}(\mathfrak{X})$ the set of all sesquilinear forms $\Omega$ on $\mathfrak{X} \times \mathfrak{X}$ with the following properties:

(i) $\Omega(x, x) \geq 0 \quad \forall x \in \mathfrak{X}$;

(ii) $\Omega(x a, b)=\Omega\left(a, x^{*} b\right) \quad \forall x \in \mathfrak{X}, \forall a, b \in \mathfrak{A}_{0}$;

(iii) $|\Omega(x, y)| \leq p(x) p(y)$ for some $\tau$-continuous seminorm $p$ on $\mathfrak{X}$ and all $x, y \in \mathfrak{X}$;

(iv) $\Omega(e, e) \leq 1$.

The locally convex quasi $\mathrm{C}^{*}$-algebra $\left(\mathfrak{X}, \mathfrak{A}_{0}\right)$ is called ${ }^{*}$-semisimple if $x \in \mathfrak{X}, \Omega(x, x)=$ 0 , for every $\Omega \in \mathcal{S}(\mathfrak{X})$, implies $x=0$.

We denote by $\mathcal{T}(\mathfrak{X}) \subseteq \mathcal{S}(\mathfrak{X})$ the set of all sesquilinear forms $\Omega \in \mathcal{S}(\mathfrak{X})$ with the following property

(iv) $\Omega(x, x)=\Omega\left(x^{*}, x^{*}\right), \forall x \in \mathfrak{X}$.

Remark 2.2. Notice that

- By (iv) of Definition 2.1 and by polarization, we get

$$
\Omega\left(y^{*}, x^{*}\right)=\Omega(x, y), \quad \forall x, y \in \mathfrak{X} .
$$

- The set $\mathcal{T}(\mathfrak{X})$ is convex.

Example 2.3. Let $\mathfrak{M}$ be a von Neumann algebra and $\varphi$ a normal faithful semifinite trace on $\mathfrak{M}_{+}$. Then, $\left(L^{p}(\varphi), \mathcal{J}_{p}\right), p \geq 2$, is a ${ }^{*}$-semisimple Banach quasi ${ }^{*}$-algebra. If $\varphi$ is a finite trace (we assume $\varphi(\mathbb{I})=1$ ), then $\left(L^{p}(\varphi), \mathfrak{M}\right.$ ), with $p \geq 2$, is a *semisimple locally convex quasi $\mathrm{C}^{*}$-algebra. If $p \geq 2, L^{p}$-spaces possess a sufficient family of positive sesquilinear forms. Indeed, in this case, since for every $W \in L^{p}(\varphi)$, $|W|^{p-2} \in L^{p /(p-2)}(\varphi)$, then the sesquilinear form $\Omega_{W}$ defined by

$$
\Omega_{W}(X, Y):=\frac{\varphi\left[X\left(Y|W|^{p-2}\right)^{*}\right]}{\|W\|_{p, \varphi}^{p-2}}
$$

is positive and satisfies the conditions (i),(ii),(iii) and (iv) of Definition 2.1, (see [9], and 24] for more details). Moreover,

$$
\Omega_{W}(W, W)=\|W\|_{p, \varphi}^{p} .
$$

\footnotetext{
${ }^{1}$ In previous papers this structure was called proper $\mathrm{CQ}^{*}$-algebra. Since this is the sole case we consider here we will systematically omit the specification proper.
} 
Remark 2.4. The notion of *-semisimplicity of locally convex partial *-algebras has been studied in full generality in [2] and [14].

Definition 2.5. Let $\mathfrak{M}$ be a von Neumann algebra and $\varphi$ a normal faithful semifinite trace defined on $\mathfrak{M}_{+}$. We denote by $L_{\text {loc }}^{p}(\varphi)$ the set of all measurable operators $T$ such that $T P \in L^{p}(\varphi)$, for every central $\varphi$-finite projection $P$ of $\mathfrak{M}$.

Remark 2.6. The von Neumann algebra $\mathfrak{M}$ is a subset of $L_{\mathrm{loc}}^{p}(\varphi)$. Indeed, if $X \in \mathfrak{M}$, then for every $\varphi$-finite central projection $P$ of $\mathfrak{M}$ the product $X P$ belongs to the ${ }^{*}$-ideal $\mathcal{J}_{p}$.

Throughout this section we are given a von Neumann algebra $\mathfrak{M}$ on a Hilbert space $\mathcal{H}$ with a family $\left\{P_{j}\right\}_{j \in J}$ of $\varphi$-finite central projections of $\mathfrak{M}$ such that

- if $l, m \in J, l \neq m$, then $P_{l} P_{m}=0$ (i.e., the $P_{j}$ 's are orthogonal);

- $\bigvee_{j \in J} P_{j}=\mathbb{I}$; where $\bigvee_{j \in J} P_{j}$ denotes the projection onto the subspace generated by $\left\{P_{j} \mathcal{H}: j \in J\right\}$.

The previous two conditions are always realized in a von Neumann $\mathfrak{M}$ algebra with a faithful normal semifinite trace (see Lemma 1.1] and [15, 20] for more details).

If $\varphi$ is a normal faithful semifinite trace on $\mathfrak{M}_{+}$, we define, for each $X \in \mathfrak{M}$, the following seminorms $q_{j}(X):=\left\|X P_{j}\right\|_{p, \varphi}$ on $\mathfrak{M}, j \in J$. The traslation-invariant locally convex topology defined by the system $\left\{q_{j}: j \in J\right\}$ is denoted by $\tau_{p}$.

Definition 2.7. Let $\mathfrak{M}$ be a von Neumann algebra and $\varphi$ a normal faithful semifinite trace defined on $\mathfrak{M}_{+}$. We denote by $\widetilde{\mathfrak{M}}^{\tau_{p}}$ the $\tau_{p}$ completion of $\mathfrak{M}$.

Proposition 2.8. Let $\mathfrak{M}$ be a von Neumann algebra and $\varphi$ a normal faithful semifinite trace on $\mathfrak{M}_{+}$. Then $L_{\mathrm{loc}}^{p}(\varphi) \subseteq \widetilde{\mathfrak{M}}^{\tau_{p}}$. Moreover, if there exists a family $\left\{P_{j}\right\}_{j \in J}$ as above, where all $P_{j}^{\prime}$ 's are mutually equivalent, then $L_{\mathrm{loc}}^{p}(\varphi)=\widetilde{\mathfrak{M}}^{\tau_{p}}$.

Proof. From Remark 2.6, $\mathfrak{M} \subseteq L_{\mathrm{loc}}^{p}(\varphi)$. If $Y \in L_{\text {loc }}^{p}(\varphi)$, for every $j \in J$ we have $Y P_{j} \in$ $L^{p}(\varphi)$. Then, for every $j \in J$, there exist $\left(X_{n}^{j}\right)_{n=1}^{\infty} \subseteq \mathcal{J}_{p}$ such that $\left\|X_{n}^{j}-Y P_{j}\right\|_{p, \varphi} \underset{n \rightarrow \infty}{\longrightarrow} 0$.

Let $\mathbb{F}_{J}$ be the family of finite subsets of $J$ ordered by inclusion and $F \in \mathbb{F}_{J}$.

We put

$$
T_{n, F}:=\sum_{j \in F} X_{n}^{j} P_{j} \in \mathfrak{M}
$$

Then the net $\left(T_{n, F}\right)$ converges to $Y$ with respect to $\tau_{p}$. Indeed, for every $m \in J$,

$$
q_{m}\left(T_{n, F}-Y\right)=\left\|\left(T_{n, F}-Y\right) P_{m}\right\|_{p, \varphi}=\left\|\left(X_{n}^{m}-Y\right) P_{m}\right\|_{p, \varphi}
$$

for sufficiently large $F$. Thus, the inequality $\left\|\left(X_{n}^{m}-Y\right) P_{m}\right\|_{p, \varphi} \leq\left\|X_{n}^{m}-Y P_{m}\right\|_{p, \varphi}$ implies that $q_{m}\left(T_{n, F}-Y\right) \underset{n, F}{\longrightarrow} 0$.

Hence $L_{\mathrm{loc}}^{p}(\varphi) \subseteq \widetilde{\mathfrak{M}}^{\tau_{p}}$.

On the other hand, assume that all $P_{j}$ 's are mutually equivalent. Then, if $Y \in \widetilde{\mathfrak{M}}^{\tau_{p}}$, there exists a net $\left(X_{\alpha}\right) \subseteq \mathfrak{M}$ such that $X_{\alpha} \rightarrow Y$ with respect to $\tau_{p}$; hence

$$
X_{\alpha} P_{j} \rightarrow Y P_{j} \in L^{p}(\varphi) \text { in }\|\cdot\|_{p, \varphi} .
$$


But for each central $\varphi$-finite projection $P$ we have

$$
\varphi(P)=\varphi\left(P \sum_{j \in J} P_{j}\right)=\sum_{j \in J} \varphi\left(P P_{j}\right) .
$$

By our assumption, for any $l, m \in J$, we may pick $U \in \mathfrak{M}$ so that $U^{*} U=P_{l}$ and $U U^{*}=P_{m}$, hence,

$$
\varphi\left(P P_{l}\right)=\varphi\left(P U^{*} U\right)=\varphi\left(U P U^{*}\right)=\varphi\left(P U U^{*}\right)=\varphi\left(P P_{m}\right) .
$$

So, all terms on the right hand side of (2) are equal and since the above series converges, only a finite number of them can be nonzero. Thus, for some $s \in \mathbb{N}$ we may write $J=\{1, \ldots, s\}$ and then

$$
P=P \sum_{j \in J} P_{j}=P \sum_{j=1}^{s} P_{j}=\sum_{j=1}^{s} P P_{j}
$$

and hence

$$
Y P=\sum_{j=1}^{s} Y P P_{j}=\sum_{j=1}^{s} Y P_{j} P \in L^{p}(\varphi) .
$$

Therefore, if $Y \in \widetilde{\mathfrak{M}}^{\tau_{p}}$, for each central $\varphi$-finite projection $P$, we have $Y P \in L^{p}(\varphi)$. Hence $L_{\mathrm{loc}}^{p}(\varphi) \supseteq \widetilde{\mathfrak{M}}^{\tau_{p}}$.

Remark 2.9. In general it is not guaranteed that a von Neumann algebra possesses an orthogonal family $\left\{P_{j}\right\}_{j \in J}$ of mutually equivalent finite central projection such that $\bigvee_{j \in J} P_{j}=\mathbb{I}$, but, if this is the case, then $L_{\text {loc }}^{p}(\varphi)=\widetilde{\mathfrak{M}}^{\tau_{p}}$.

Theorem 2.10. Let $\mathfrak{M}$ be a von Neumann algebra on a Hilbert space $\mathcal{H}$ and $\varphi$ a normal faithful semifinite trace on $\mathfrak{M}_{+}$. Then $\left(\widetilde{\mathfrak{M}}^{\tau_{p}}, \mathfrak{M}\right)$ is a locally convex quasi $C^{*}$-algebra with respect to $\tau_{p}$, consisting of measurable operators.

Proof. The topology $\tau_{p}$ satisfies the properties $\left(\mathrm{T}_{1}\right)-\left(\mathrm{T}_{4}\right)$. We just prove here $\left(\mathrm{T}_{3}\right)-\left(\mathrm{T}_{4}\right)$.

- $\left(\mathrm{T}_{3}\right)$ For each $\lambda \in J$,

$$
q_{\lambda}(X Y)=\left\|P_{\lambda} X Y\right\|_{p, \varphi} \leq\|X\|\left\|P_{\lambda} Y\right\|_{p, \varphi}=\|X\| q_{\lambda}(Y), \quad \forall X, Y \in \mathfrak{M} ;
$$

- $\left(\mathrm{T}_{4}\right)$ The set $\mathcal{U}(\mathfrak{M})_{+}:=\left\{X \in(\mathfrak{M})_{+}:\|X\| \leq 1\right\}$ is $\tau_{p^{-}}$closed. To see this consider a net $\left\{F_{\alpha}\right\}$ in $\mathcal{U}(\mathfrak{M})_{+}: F_{\alpha} \rightarrow F$ in the topology $\tau_{p}$, then for each $j \in J$, we have $\left\|\left(F_{\alpha}-F\right) P_{j}\right\|_{p, \varphi} \rightarrow 0$. By assumption on $P_{j}$, the trace $\varphi$ is a normal faithful finite trace on the von Neumann algebra on $P_{j} \mathfrak{M}_{+}$. Then (see [9]) $\left(L^{p}(\varphi), P_{j} \mathfrak{M}\right)$ is a $\mathrm{CQ}^{*}$-algebra. Therefore, using $\left(\mathrm{T}_{4}\right)$ for $\left(L^{p}(\varphi), P_{j} \mathfrak{M}\right)$, we have $F P_{j} \in \mathcal{U}\left(P_{j} \mathfrak{M}\right)_{+}$for each $j \in J$. This, by definition, implies that $F \in \mathfrak{M}$. Indeed, for every

$$
h=\sum_{j \in J} P_{j} h \in \mathcal{H}=\bigoplus_{j \in J} P_{j} \mathcal{H}
$$


we have

$$
\begin{aligned}
\|F h\|_{\mathcal{H}}^{2} & =\sum_{j \in J}\left\|F P_{j} h\right\|^{2} \\
& =\sum_{j \in J}\left\|F P_{j} P_{j} h\right\|^{2} \leq \sum_{j \in J}\left\|P_{j} h\right\|^{2} \\
& =\|h\|_{\mathcal{H}}^{2} .
\end{aligned}
$$

Hence $F \in \mathcal{U}(\mathfrak{M})_{+}$.

Remark 2.11. By Proposition 2.8, $\left(L_{\mathrm{loc}}^{p}(\varphi), \mathfrak{M}\right)$ itself is a locally convex quasi $C^{*}$ algebra with respect to $\tau_{p}$.

\section{The RePRESEntATion theOREMS}

Let $\left(\mathfrak{X}, \mathfrak{A}_{0}\right)$ be a locally convex quasi $\mathrm{C}^{*}$-algebra with a unit $e$. For each $\Omega \in \mathcal{T}(\mathfrak{X})$, we define a linear functional $\omega_{\Omega}$ on $\mathfrak{A}_{0}$ by

$$
\omega_{\Omega}(a):=\Omega(a, e) \quad a \in \mathfrak{A}_{0} .
$$

We have

$$
\omega_{\Omega}\left(a^{*} a\right)=\Omega\left(a^{*} a, e\right)=\Omega(a, a)=\Omega\left(a^{*}, a^{*}\right)=\omega_{\Omega}\left(a a^{*}\right) \geq 0 .
$$

This shows at once that $\omega_{\Omega}$ is positive and tracial.

By the Gelfand-Naimark theorem each $C^{*}$-algebra is isometrically ${ }^{*}$-isomorphic to a $C^{*}$-algebra of bounded operators in Hilbert space. This isometric ${ }^{*}$-isomorphism is called the universal ${ }^{*}$-representation. We denote it by $\pi$.

For every $\Omega \in \mathcal{T}(\mathfrak{X})$ and $a \in \mathfrak{A}_{0}$, we put

$$
\varphi_{\Omega}(\pi(a))=\omega_{\Omega}(a) .
$$

Then, for each $\Omega \in \mathcal{T}(\mathfrak{X}), \varphi_{\Omega}$ is a positive bounded linear functional on the operator algebra $\pi\left(\mathfrak{A}_{0}\right)$.

Clearly,

$$
\varphi_{\Omega}(\pi(a))=\omega_{\Omega}(a)=\Omega(a, e) .
$$

Using the fact that the family $\left\{p_{\lambda}\right\}$ is directed, we conclude that there exist $\gamma>0$ and $\lambda \in \Lambda$ such that

$$
\left|\varphi_{\Omega}(\pi(a))\right|=\left|\omega_{\Omega}(a)\right|=|\Omega(a, e)| \leq \gamma^{2} p_{\lambda}(a e) p_{\lambda}(e) .
$$

Then, by $\left(\mathrm{T}_{3}\right)$, for some $\lambda^{\prime} \in \Lambda$

$$
\left|\varphi_{\Omega}(\pi(a))\right| \leq \gamma^{2}\|a\|_{0} p_{\lambda^{\prime}}(e)^{2}
$$

Thus $\varphi_{\Omega}$ is continuous on $\pi\left(\mathfrak{A}_{0}\right)$.

By [15, Vol. 2, Proposition 10.1.1], $\varphi_{\Omega}$ is weakly continuous and so it extends uniquely to $\pi\left(\mathfrak{A}_{0}\right)^{\prime \prime}$, by the Hahn-Banach theorem. Moreover, since $\varphi_{\Omega}$ is a trace on $\pi\left(\mathfrak{A}_{0}\right)$, the extension $\widetilde{\varphi_{\Omega}}$ is also a trace on the von Neumann algebra $\mathfrak{M}:=\pi\left(\mathfrak{A}_{0}\right)^{\prime \prime}$ generated by $\pi\left(\mathfrak{A}_{0}\right)$.

Clearly, the set $\mathfrak{N}_{\mathcal{T}}\left(\mathfrak{A}_{0}\right)=\left\{\widetilde{\varphi_{\Omega}} ; \Omega \in \mathcal{T}(\mathfrak{X})\right\}$ is convex. 
Definition 3.1. The locally convex quasi $\mathrm{C}^{*}$-algebra $\left(\mathfrak{X}, \mathfrak{A}_{0}\right)$ is said to be strongly *-semisimple if

(a) the equality $\Omega(x, x)=0$, for every $\Omega \in \mathcal{T}(\mathfrak{X})$, implies $x=0$;

(b) the set $\mathfrak{N}_{\mathcal{T}}\left(\mathfrak{A}_{0}\right)$ is $w^{*}$-closed.

Remark 3.2. If $\left(\mathfrak{X}, \mathfrak{A}_{0}\right)$ is a $C Q^{*}$-algebra, by [9, Proposition 4.1], (b) is automatically satisfied.

Example 3.3. Let $\mathfrak{M}$ be a von Neumann algebra and $\varphi$ a normal faithful semifinite trace on $\mathfrak{M}_{+}$. Then, as seen in Example 2.3, if $\varphi$ is a finite trace, then $\left(L^{p}(\varphi), \mathfrak{M}\right)$, with $p \geq 2$, is a ${ }^{*}$-semisimple locally convex quasi $\mathrm{C}^{*}$-algebra. The conditions (a) and (b) of Definition 3.1 are satisfied. Indeed, in this case, the set $\mathfrak{N}_{\mathcal{T}}(\mathfrak{M})$ is $w^{*}$-closed by [9, Proposition 4.1]. Therefore $\left(L^{p}(\varphi), \mathfrak{M}\right)$, with $\varphi$ finite, is a strongly ${ }^{*}$-semisimple locally convex quasi $\mathrm{C}^{*}$-algebra .

Let $\left(\mathfrak{X}, \mathfrak{A}_{0}\right)$ be a locally convex quasi $\mathrm{C}^{*}$-algebra with unit $e, \pi$ the universal representation of $\mathfrak{A}_{0}$ and $\mathfrak{M}=\pi\left(\mathfrak{A}_{0}\right)^{\prime \prime}$. Denote by $\|f\|^{\sharp}$ the norm of a bounded functional $f$ on $\mathfrak{M}$ and by $\mathfrak{M}^{\sharp}$ the topological dual of $\mathfrak{M}$ then, the norm $\left\|\widetilde{\varphi_{\Omega}}\right\|^{\sharp}$ of $\widetilde{\varphi_{\Omega}}$ as a linear functional on $\mathfrak{M}$ equals the norm of $\varphi_{\Omega}$ as a functional on $\pi\left(\mathfrak{A}_{0}\right)$.

By (iv) of Definition 2.1, $\left\|\widetilde{\varphi_{\Omega}}\right\|^{\sharp}=\widetilde{\varphi}_{\Omega}(\pi(e))=\Omega(e, e) \leq 1$.

Hence, if (b) of Definition 3.1 is satisfied, the set $\mathfrak{N}_{\mathcal{T}}\left(\overline{\mathfrak{A}}_{0}\right)$, being a $w^{*}$-closed subset of the unit ball of $\mathfrak{M}^{\sharp}$, is $w^{*}$-compact.

Let $\mathfrak{E N}_{\mathcal{T}}\left(\mathfrak{A}_{0}\right)$ be the set of extreme points of $\mathfrak{N}_{\mathcal{T}}\left(\mathfrak{A}_{0}\right)$; then $\mathfrak{N}_{\mathcal{T}}\left(\mathfrak{A}_{0}\right)$ coincides with $w^{*}$-closure of the convex hull of $\mathfrak{E N}_{\mathcal{T}}\left(\mathfrak{A}_{0}\right)$. Let $\pi: \mathfrak{A}_{0} \hookrightarrow \mathcal{B}(\mathcal{H})$ be its universal representation.

Then $\mathfrak{E N}_{\mathcal{T}}\left(\mathfrak{A}_{0}\right)$ is a family of normal finite traces on the von Neumann algebra $\mathfrak{M}$.

We put $\mathcal{F}:=\left\{\Omega \in \mathcal{T}(\mathfrak{X}) ; \widetilde{\varphi_{\Omega}} \in \mathfrak{E N}_{\mathcal{T}}\left(\mathfrak{A}_{0}\right)\right\}$ and denote by $P_{\Omega}$ the support projection corresponding to the trace $\widetilde{\varphi_{\Omega}}$. By [9, Lemma 3.5], $\left\{P_{\Omega}\right\}_{\Omega \in \mathcal{F}}$ consists of mutually orthogonal projections and if $Q:=\bigvee_{\Omega \in \mathcal{F}} P_{\Omega}$ then

$$
\mu=\sum_{\widetilde{\varphi_{\Omega}} \in \mathfrak{E N}_{\mathcal{T}}\left(\mathfrak{A}_{0}\right)} \widetilde{\varphi_{\Omega}}
$$

is a normal faithful semifinite trace defined on the direct sum (see [20] and [26]) of von Neumann algebras

$$
Q \mathfrak{M}=\bigoplus_{\Omega \in \mathcal{F}} P_{\Omega} \mathfrak{M}
$$

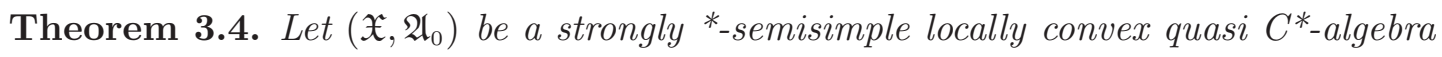
with unit e, $\pi$ the universal representation of $\mathfrak{A}_{0}$. Then there exists a monomorphism

$$
\Phi: x \in \mathfrak{X} \rightarrow \Phi(x):=\widetilde{X} \in \widetilde{Q \mathfrak{M}}^{\tau_{2}}
$$

with the following properties:

(i) $\Phi$ extends the isometry $\pi: \mathfrak{A}_{0} \hookrightarrow \mathcal{B}(\mathcal{H})$ given by the Gelfand-Naimark theorem;

(ii) $\Phi\left(x^{*}\right)=\Phi(x)^{*}$, for every $x \in \mathfrak{X}$;

(iii) $\Phi(x y)=\Phi(x) \Phi(y)$, for all $x, y \in \mathfrak{X}$ such that $x \in \mathfrak{A}_{0}$ or $y \in \mathfrak{A}_{0}$. 
Proof. Let $\left\{p_{\lambda}\right\}_{\lambda \in \Lambda}$ be, as before, the family of seminorms defining the topology $\tau$ of $\mathfrak{X}$. For fixed $x \in \mathfrak{X}$, there exists a net $\left\{a_{\alpha} ; \alpha \in \Delta\right\}$ of elements of $\mathfrak{A}_{0}$ such that $p_{\lambda}\left(a_{\alpha}-x\right) \rightarrow 0$ for each $\lambda \in \Lambda$. We put $X_{\alpha}=\pi\left(a_{\alpha}\right)$.

By (iii) of Definition 2.1, for every $\Omega \in \mathcal{T}(\mathfrak{X})$, there exist $\gamma>0$ and $\lambda^{\prime} \in \Lambda$ such that for each $\alpha, \beta \in \Delta$

$$
\begin{aligned}
\left\|P_{\Omega}\left(X_{\alpha}-X_{\beta}\right)\right\|_{2, \widetilde{\varphi_{\Omega}}} & =\left\|P_{\Omega}\left(\pi\left(a_{\alpha}\right)-\pi\left(a_{\beta}\right)\right)\right\|_{2, \widetilde{\varphi_{\Omega}}} \\
& =\left[\widetilde{\varphi_{\Omega}}\left(\left|P_{\Omega}\left(\pi\left(a_{\alpha}\right)-\pi\left(a_{\beta}\right)\right)\right|^{2}\right)\right]^{1 / 2}= \\
& =\left[\Omega\left(\left(a_{\alpha}-a_{\beta}\right)^{*}\left(a_{\alpha}-a_{\beta}\right), e\right)\right]^{1 / 2} \\
& =\left[\Omega\left(a_{\alpha}-a_{\beta}, a_{\alpha}-a_{\beta}\right)\right]^{1 / 2} \leq \gamma p_{\lambda^{\prime}}\left(a_{\alpha}-a_{\beta}\right) \underset{\alpha, \beta}{\longrightarrow} 0 .
\end{aligned}
$$

Let $\widetilde{X_{\Omega}}$ be the $\|\cdot\|_{2, \widetilde{\varphi_{\Omega}}}$-limit of the net $\left(P_{\Omega} X_{\alpha}\right)$ in $L^{2}\left(\widetilde{\varphi_{\Omega}}\right)$. Clearly $\widetilde{X_{\Omega}}=P_{\Omega} \widetilde{X_{\Omega}}$ We define

$$
\Phi(x):=\sum_{\Omega \in \mathcal{F}} P_{\Omega} \widetilde{X_{\Omega}}=: \widetilde{X}
$$

Clearly $\tilde{X} \in \widetilde{Q \mathfrak{M}}^{\tau_{2}}$.

It is easy to see that the map $x \ni \mathfrak{X} \mapsto \widetilde{X} \in \widetilde{Q M}^{\tau_{2}}$ is well defined and injective. Indeed, if $a_{\alpha} \rightarrow 0$, there exist $\gamma>0$ and $\lambda^{\prime} \in \Lambda$ such that

$$
\begin{aligned}
\left\|P_{\Omega} X_{\alpha}\right\|_{2, \widetilde{\varphi_{\Omega}}} & =\left\|P_{\Omega} \pi\left(a_{\alpha}\right)\right\|_{2, \widetilde{\varphi_{\Omega}}} \\
& =\left[\widetilde{\varphi_{\Omega}}\left(\mid P_{\Omega}\left(\left.\pi\left(a_{\alpha}\right)\right|^{2}\right)\right]^{1 / 2}=\right. \\
& =\left[\Omega\left(a_{\alpha}^{*} a_{\alpha}, e\right)\right]^{1 / 2} \\
& =\left[\Omega\left(a_{\alpha}, a_{\alpha}\right)\right]^{1 / 2} \leq \gamma p_{\lambda^{\prime}}\left(a_{\alpha}\right) \rightarrow 0 .
\end{aligned}
$$

Thus $P_{\Omega}\left(X_{\alpha}\right)=0$ for every $\Omega \in \mathcal{T}(\mathfrak{X})$, then $\tilde{X}=0$. Moreover if $P_{\Omega} \widetilde{X}=0$, for each $\Omega \in \mathcal{F}$, then $\Omega(x, x)=0$ for every $\Omega \in \mathcal{F}$. Since every $\Omega \in \mathcal{T}(\mathfrak{X})$ is a $\mathrm{w}^{*}$-limit of of convex combinations of elements of $\mathcal{F}$, we get $\Omega(x, x)=0$ for every $\Omega \in \mathcal{T}(\mathfrak{X})$. Therefore, by assumption, $x=0$.

\section{Remark 3.5.}

In the same way we can prove that

- If $\left(\mathfrak{X}, \mathfrak{A}_{0}\right)$ is a strongly *-semisimple locally convex quasi $\mathrm{C}^{*}$-algebra and there exists a faithful $\Omega \in \mathcal{T}(\mathfrak{X})$ (i.e., the equality $\Omega(x, x)=0$, implies $x=0$ ) then there exists a monomorphism

$$
\Phi: x \in \mathfrak{X} \rightarrow \Phi(x):=\widetilde{X} \in L^{2}\left(\widetilde{\varphi_{\Omega}}\right)
$$

with the following properties:

(i) $\Phi$ extends the isometry $\pi: \mathfrak{A}_{0} \hookrightarrow \mathcal{B}(\mathcal{H})$ given by the Gelfand-Naimark theorem;

(ii) $\Phi\left(x^{*}\right)=\Phi(x)^{*}$, for every $x \in \mathfrak{X}$

(iii) $\Phi(x y)=\Phi(x) \Phi(y)$, for all $x, y \in \mathfrak{X}$ such that $x \in \mathfrak{A}_{0}$ or $y \in \mathfrak{A}_{0}$. 
- If the semifinite von Neumann algebra $\pi\left(\mathfrak{A}_{0}\right)^{\prime \prime}$ admits an orthogonal family of mutually equivalent projections $\left\{P_{i}^{\prime} ; i \in I\right\}$ such that $\sum_{i \in I} P_{i}^{\prime}=\mathbb{I}$, then is easy to see that the map $x \in \mathfrak{X} \rightarrow \widetilde{X} \in L_{l o c}^{2}(\tau)$ is a monomorphism.

Acknowledgement. The authors wish to express their gratitude to the referee for pointing out several inaccuracies in a previous version of the paper and for his/her fruitful comments.

\section{REFERENCES}

1. J.-P. Antoine, A.Inoue and C.Trapani, Partial *-algebras and their Operator Realizations, Kluwer, Dordrecht, 2002.

2. J-P. Antoine, G. Bellomonte, C. Trapani, Fully representable and ${ }^{*}$-semisimple topological partial *-algebras, Studia Mathematica, No.2, 208 (2012), 167-194 DOI: 10.4064/sm208-2-4.

3. F. Bagarello, M. Fragoulopoulou, A. Inoue and C. Trapani, Locally convex quasi C ${ }^{*}$-normed algebras. J. Math. Anal. Appl., 366, (2010), no. 2, 593606.

4. F.Bagarello, A.Inoue and C.Trapani, Some classes of topological quasi *-algebras, Proc. Amer. Math. Soc., 129 (2001), 2973-2980.

5. F.Bagarello and C.Trapani,States and representations of $C Q^{*}$-algebras, Ann. Inst. H. Poincaré 61, 103-133(1994).

6. F.Bagarello and C.Trapani, $L^{p}$-spaces as quasi *-algebras, J.Math. Anal. Appl. 197, 810-824 (1996).

7. F.Bagarello and C.Trapani, $C Q^{*}$-algebras: structure properties Publ. RIMS, Kyoto Univ. 32, 85116 (1996).

8. F.Bagarello and C.Trapani, Morphisms of certain Banach $C^{*}$-modules, Publ. RIMS, Kyoto Univ. 36 (2000) 681-705

9. F. Bagarello, C. Trapani and S. Triolo, Quasi *-algebras of measurable operators, Studia Mathematica 172 (2006), no. 3, 289-305.

10. F. Bagarello, C. Trapani and S. Triolo, A note on faithful traces on a von Neumann algebra, Rend. Circ. Mat. Palermo 55 (2006), no. 1, 21-28.

11. F. Bagarello, C. Trapani, S. Triolo; Representable states on quasi-local quasi *-algebras, J. Math. Phys. 52 (2011), no. 1, 013510, 11 pp.

12. B. Bongiorno, C. Trapani, S. Triolo Extensions of positive linear functionals on a topological *algebra, Rocky Mt. J. Math. 40(6), 17451777 (2010).

13. M. Fragoulopoulou, A. Inoue and K.-D. Kürsten, On the completion of a $C^{*}$-normed algebra under a locally convex algebra topology, Contemporary Math. Vol.5, 427; 155-166 (2007).

14. M. Fragoulopoulou, C. Trapani, C. and S. Triolo, Locally convex quasi *-algebras with sufficiently many *-representations, J.Math. Anal. Appl., 388 (2012), 1180-1193 .

15. R. V. Kadison and J. R. Ringrose, Fundamentals of the Theory of Operator Algebras, Vol. 1-4, Academic Press, Orlando, London, 1986.

16. C. La Russa and S. Triolo; Radon - Nikodym theorem in topological quasi *algebras. J. Operator Th. 69, (2013) 423-433.

17. E. Nelson, Notes on noncommutative integration, J. Funct. Anal., 15 (1974) 103-116

18. K. Schmüdgen, Unbounded Operator Algebras and Representation Theory, Akademie-Verlag, Berlin, 1990.

19. I. E. Segal, A noncommutative extension of abstract integration, Ann. Math. 57 (1953), 401-457.

20. M. Takesaki, Theory of Operator Algebras.I, Springer-Verlag, New York, 1979.

21. C.Trapani, $C Q^{*}$-algebras of operators and application to Quantum Models, in Proceedings of the Second ISAAC Conference, Fukuoka, Japan, Volume 1, 679-685, Kluwer (2000).

22. C. Trapani and S. Triolo, Representations of modules over a *-algebra and related seminorms, Studia Mathematica 184 (2008), 133-148.

23. C. Trapani and S. Triolo, Faithful Representations of left $C^{*}$-modules, Rend. Circolo Mat. Palermo 59 (2010), 295-302. 
24. S. Triolo, $W Q^{*}$-Algebras of measurable operators, Indian J. Pure Appl. Math. 43 (2012), 601-617.

25. S. Triolo, Moduli di Banach su $C^{*}$ algebre, Rappresentazioni Hilbertiane ed in spazi Lp non commutativi, Bollettino Umi sez. A Fascicolo N. 2 - (Agosto 2006).

26. S. Triolo, Possible extensions of the noncommutative integral, Rend. Circ. Mat. Palermo, (2) 60 (2011), no 3, 409-416. DOI 10.1007/s12215-011-0063-1.

27. S. Triolo, Extensions of the non commutative integration, submitted to Complex Analysis and Operator Theory.

Dipartimento di Matematica e Informatica, Università di Palermo, I-90123 Palermo (ITALY)

E-mail address: camillo.trapani@unipa.it

Dipartimento DEIM, Università di PAlermo, I-90123 PAlermo (ItAly)

E-mail address: salvatore.triolo@unipa.it 